\title{
Large-Scale Photochemical Reactions of Nanocrystalline Suspensions: A Promising Green Chemistry Method
}

\author{
Marcel Veerman, Marino Resendiz and Miguel A. Garcia-Garibay* \\ Department of Chemistry and Biochemistry, University of California, 405 Hilgard Ave, \\ Los Angeles, CA 90095-1569 \\ mgg@chem.ucla.edu
}

The preparation and photochemistry of dicumyl ketone (DCK) were reported previously. ${ }^{1}$ The only product observed in the solid state is dicumene (DC). The corresponding ${ }^{1} \mathrm{H}$ NMR and ${ }^{13} \mathrm{C}$ NMR spectra are shown below. As previously reported, irradiation in benzene yielded dicumene, $\alpha$-methylstyrene (MST) and isopropyl benzene (iPB).<smiles>C=C(C)[Al]CC</smiles><smiles>[14CH3]C([14CH3])[Mg]</smiles>

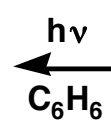

MST

iPB

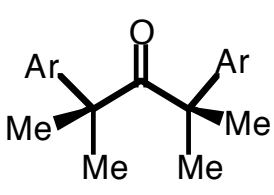

DCK

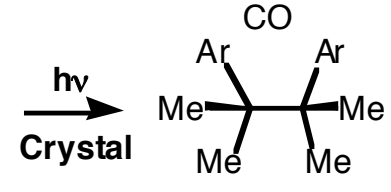

DC

\section{Photolysis}

Most photochemical reactions were carried out using a $450 \mathrm{~W}$ medium pressure AceHanovia mercury arc lamp (Catalog No. 7825-34), equipped with a circulating water cooled Pyrex jacket. Single crystals were photolyzed on the microscope state with the Pyrex $(\lambda>290 \mathrm{~nm})$ filtered output of a $1 \mathrm{~kW}$ Oriel lamp.

\section{Solution Photolysis in Benzene}

A solution of DCK (10 mg, $0.0375 \mathrm{mmol})$ in benzene $(10 \mathrm{~mL})$ was deoxygenated by bubbling argon gas through the solution for 10 minutes and sealed with a septum. The solution was positioned $10 \mathrm{~cm}$ from the lamp and was photolyzed for $6 \mathrm{~h}$. Every hour, a sample was taken and GC analyzed. 


\section{Solid-State Photolysis in Single Crystals on Crystal Films}

Single crystals grown from ethanol (ca. $1 \times 1 \times 0.5 \mathrm{~mm}^{3}$ ) were deposited on a microscope slide and exposed horizontally to the diffuse beam of $1.0 \mathrm{~kW} \mathrm{Hg-Xe} \mathrm{lamp.} \mathrm{While}$ reaction occur rapidly at the exposed surface (Figure S1), GC analysis after $10 \mathrm{~h}$ indicated that only $5 \%$ of the crystal had reacted.

Microcrystalline specimens were prepared by coating microscopes slides with a $0.1 \mathrm{M}$ ether solution of DCK and allowing the solvent to evaporate. For irradiation, the slides were positioned $10 \mathrm{~cm}$ from a horizontally held Hanovia lamp (Figure S2). Each slide contained about 4-5 mg of the reacting ketone. Samples were taken every $2 \mathrm{~h}$ and analyzed by GC and TLC until the reaction was completed after ca. $10 \mathrm{~h}$ photolysis. The microcrystals obtained in this manner remained birefringent when analyzed under cross polarizers (Figure S2).

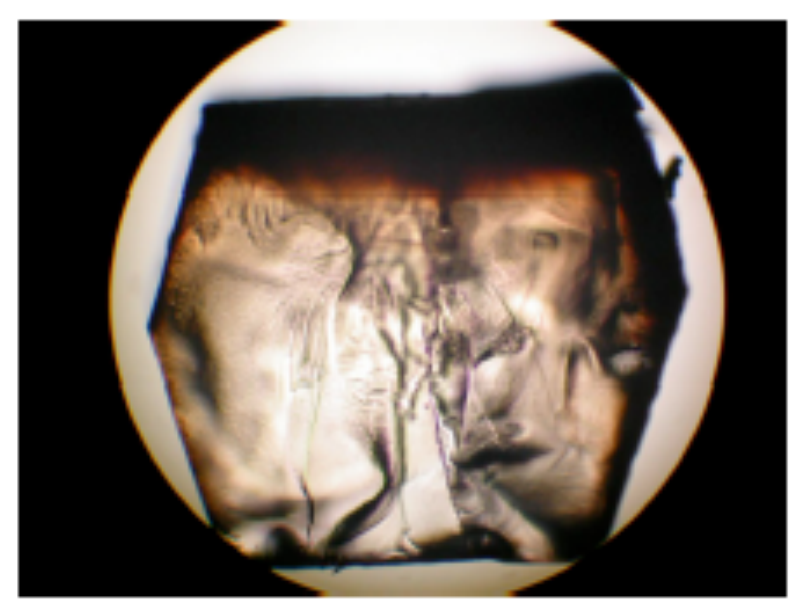

Figure S1. Heterogeneous reaction front on a crystal $\left(1.2 \times 1.3 \times 0.5 \mathrm{~mm}^{3}\right)$

1 Resendiz, M.; Garcia-Garibay, M. A., "Hammet Substituent Analysis on the Photodecarbonylation of Crystalline 1,3-Diphenylacetones" Org. Letters 2005, 7, 371374. 


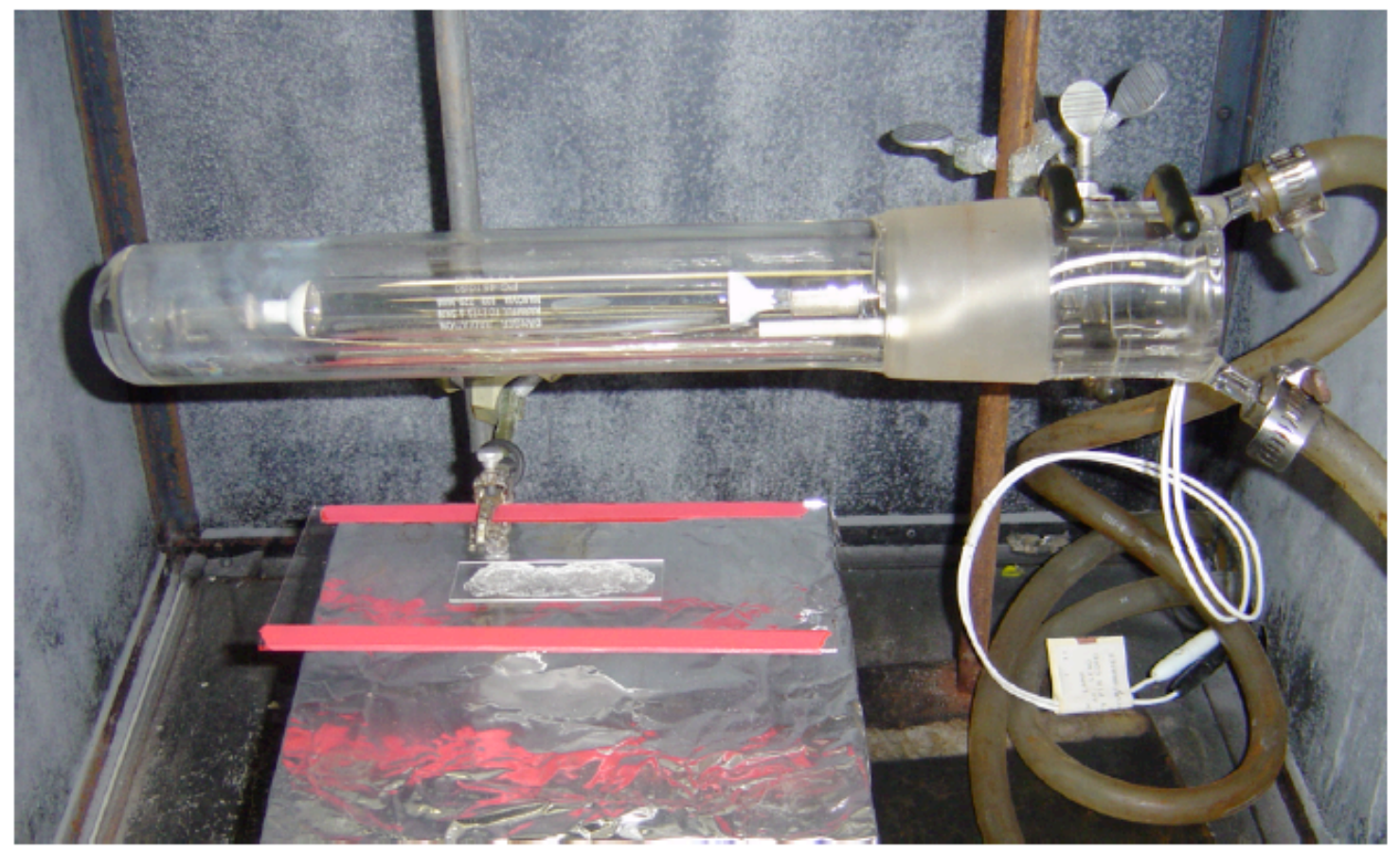

Figure S2. Irradiation of microscrystal deposited as a thin film in a microscpe slide.
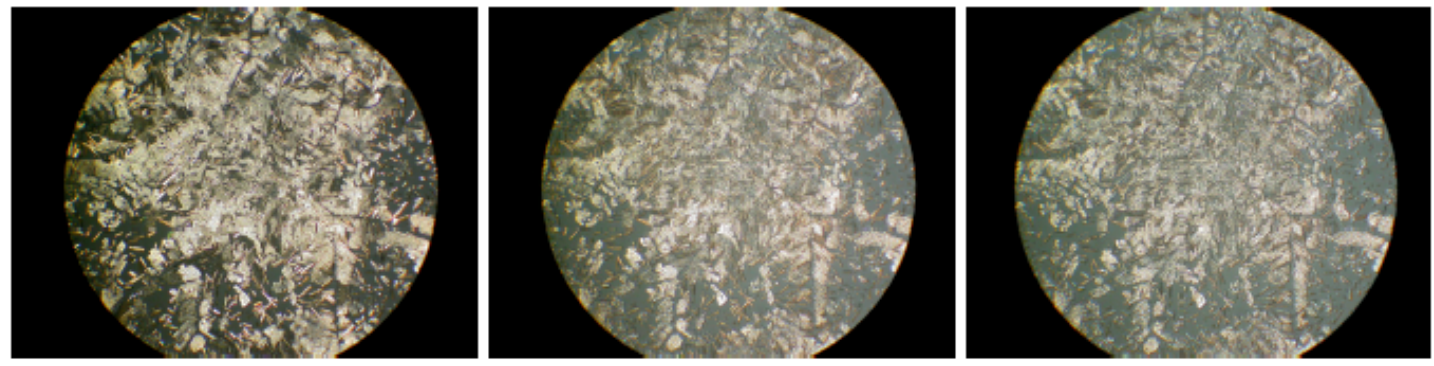

Figure S3. Thin film of microcrystals grown by ether evaporation on a microscope slide after $0 \%, 43 \%$ and $98 \%$ conversion showing birefringence under cross polarizers.

\section{Solid-State Photolysis in Dispersion of Mechanically Ground Crystals}

Crystals grown from ethanol by slow evaporation were ground with an mortar and pestle to an approximate size of $15-55 \mu \mathrm{m}$, as judged by optical microscopy. To disperse the crystals in water, the surface tension had to be reduced to prevent the agglomeration. This was accomplished by adding SDS (Figure S4). To find the optimal SDS concentration, several concentrations were tested and the minimal SDS concentration needed to form an 
suspension was ca. 1:1 mass mixture of DCK-crystals and SDS. The crystals tended to settle downward slowly and after 5-6 h, almost all crystals were on the bottom. To expose all crystals equally to the light, the suspension was magnetically stirred.
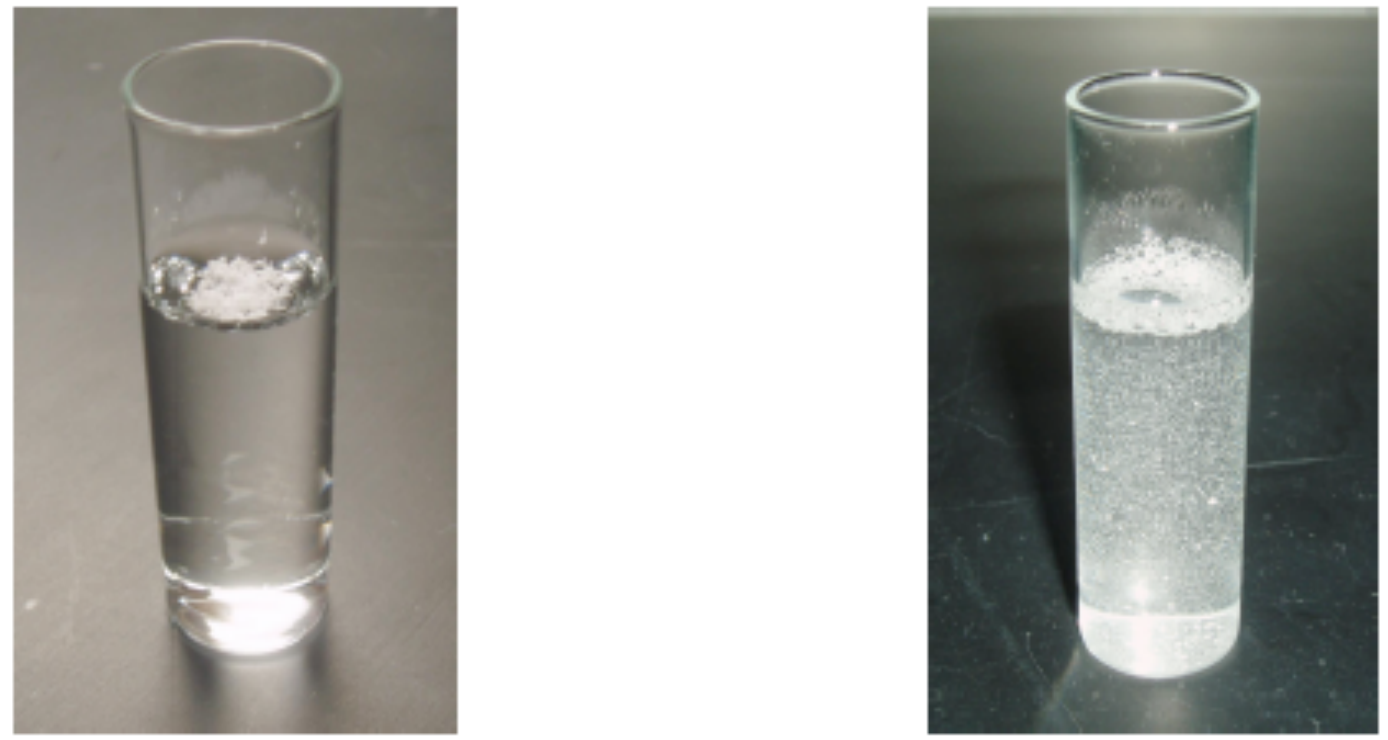

Figure S4: Samples with $10 \mathrm{mg}$ DCK crystals in $10 \mathrm{~mL}$ water, without (left) and with 10 mg of Sodium Dodecyl Sulfate (SDS).

\section{Photolysis of Crystal Suspensions.}

A $50 \mathrm{~mL}$ beaker was filled with $20 \mathrm{~mL}$ of the stirred suspension in concentrations of 2, 4, 6 and $8 \mathrm{mg} / \mathrm{mL}$ of ground DCK crystals and SDS. From the lamp to the suspension surface, the distance was set to $10 \mathrm{~cm}$ (see Figure S5). 


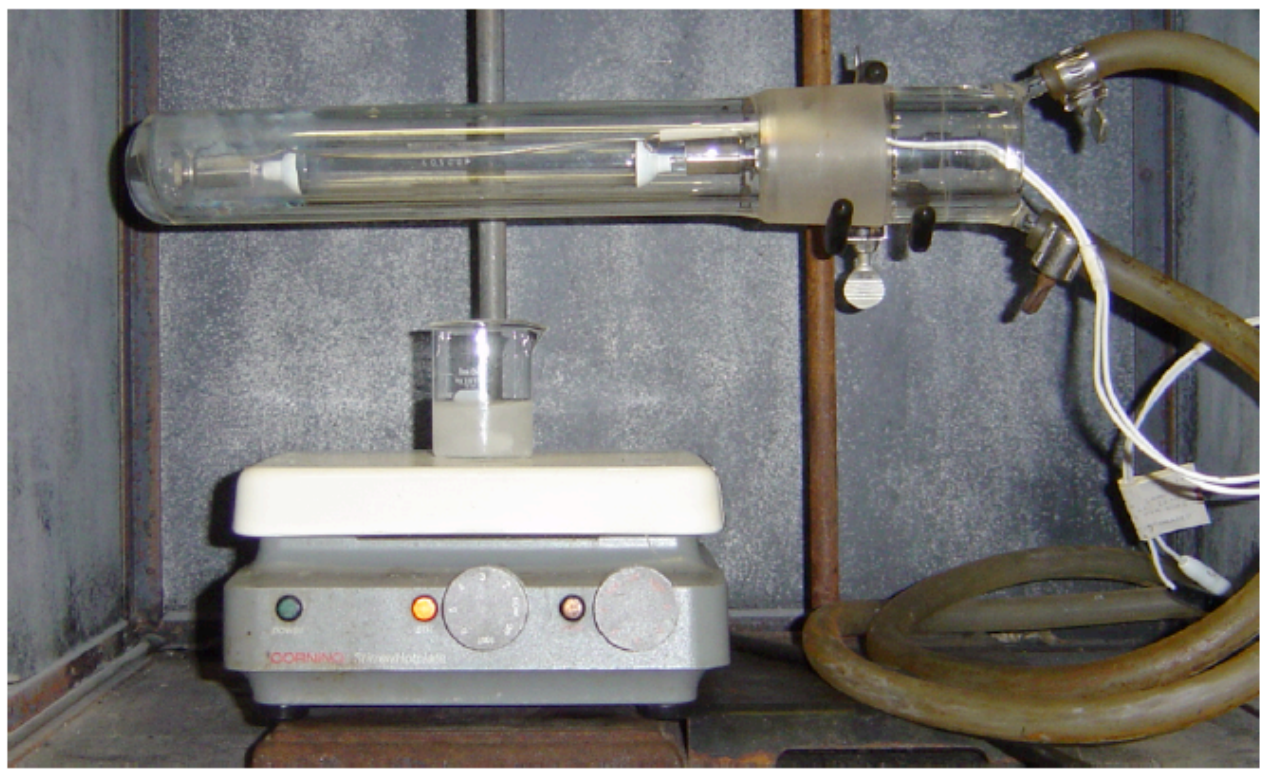

Figure S6. Experimental arrangement for irradiation of crystal suspension by an external source.

\section{Microcrystals by Precipitation. ${ }^{2}$}

Using a 1:1 mass ratio of SDS and DCK, the SDS was dissolved in a selected amount of water and kept continuously stirred while DCK was dissolved in a separate beaker in the smallest amount of acetone possible. The DCK solution was slowly added to the stirred SDS-water so that the formed crystals dispersed homogeneously. All samples were prepared with amounts of SDS above the critical micelle concentration of $10 \mathrm{mM}{ }^{3}$ To evaporate the acetone, a constant air-flow was applied over the stirred suspension the sample kept at a temperature at $25-30{ }^{\circ} \mathrm{C}$. As expected, optical microscopy revealed crystals in the range of 3.5-7.5 $\mu \mathrm{m}$ and dynamic light scattering suggested particles ranging from ca. $30 \mathrm{~nm}$ to several microns. ${ }^{4}$ FT IR analysis of the microcrystals showed

2 Kasai, H.; Nalwa, H. S.; Oikawa, H.; Okada, S.; Matsuda, H.; Minami, N.; Kakuta, A.; Ono, K.; Mukoh, A.; Nakanishi, H., Japanese Journal of Applied Physics, Part 2: Letters 1992, 31, L1132-L1134.

3 The properties of SDS were taken from the "Detergent Properties and Application" table from Aldrich; http://www.sigmaaldrich.com/img/assets/15402/Detergent Selection Table.pdf.

4 Baba, K.; Kasai, H.; Okada, S.; Oikawa, H.; Nakanishi, H. Optical Materials 2002, 21, 591-594. 
that they correspond to the same phase as macroscopic crystals obtained by slow solvent evaporation .

\section{Photolysis of 1 L Suspension with Immersion Well}

Samples with 2 and $4 \mathrm{~g} / \mathrm{L}$ of DCK and SDS were loaded in he immersion well and attached to the jacketed Hanovia lamp (Figure A10). The joints of the immersion well were sealed with needle loaded septa to avoid the formation of foam. That no UV light was coming through the suspension was determined by using a deoxygenated $0.01 \mathrm{M}$ solution of DCK in $10 \mathrm{~mL}$ benzene $(99.96 \%)$ in a Pyrex tube placed at the reactor wall.
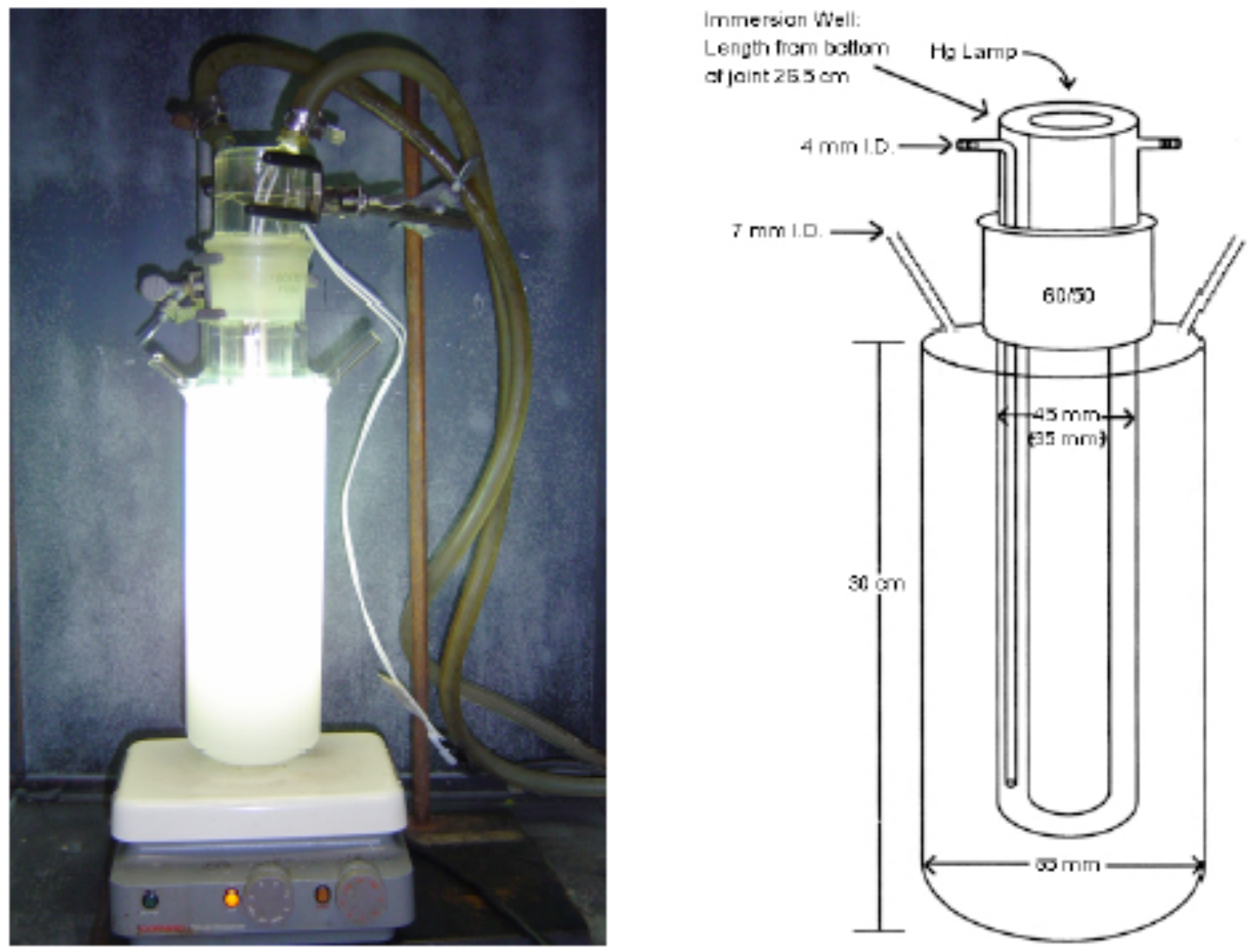

Figure S7. (Lef) Irradiation of a crystal suspension in an immersion well and (right) a schematic illustration of the same setup. 


\section{Photolysis of 3.3 L Using a Reservoir and the Immersion Well}

Using an extra $5 \mathrm{~L}$ reservoir and a circulation pump (Little Giant Pump, Cat No. 501003), the reactor could extend to a volume of $3.3 \mathrm{~L}$, and $10 \mathrm{~g}$ of microcrystals could be conversed (see Figure S8).

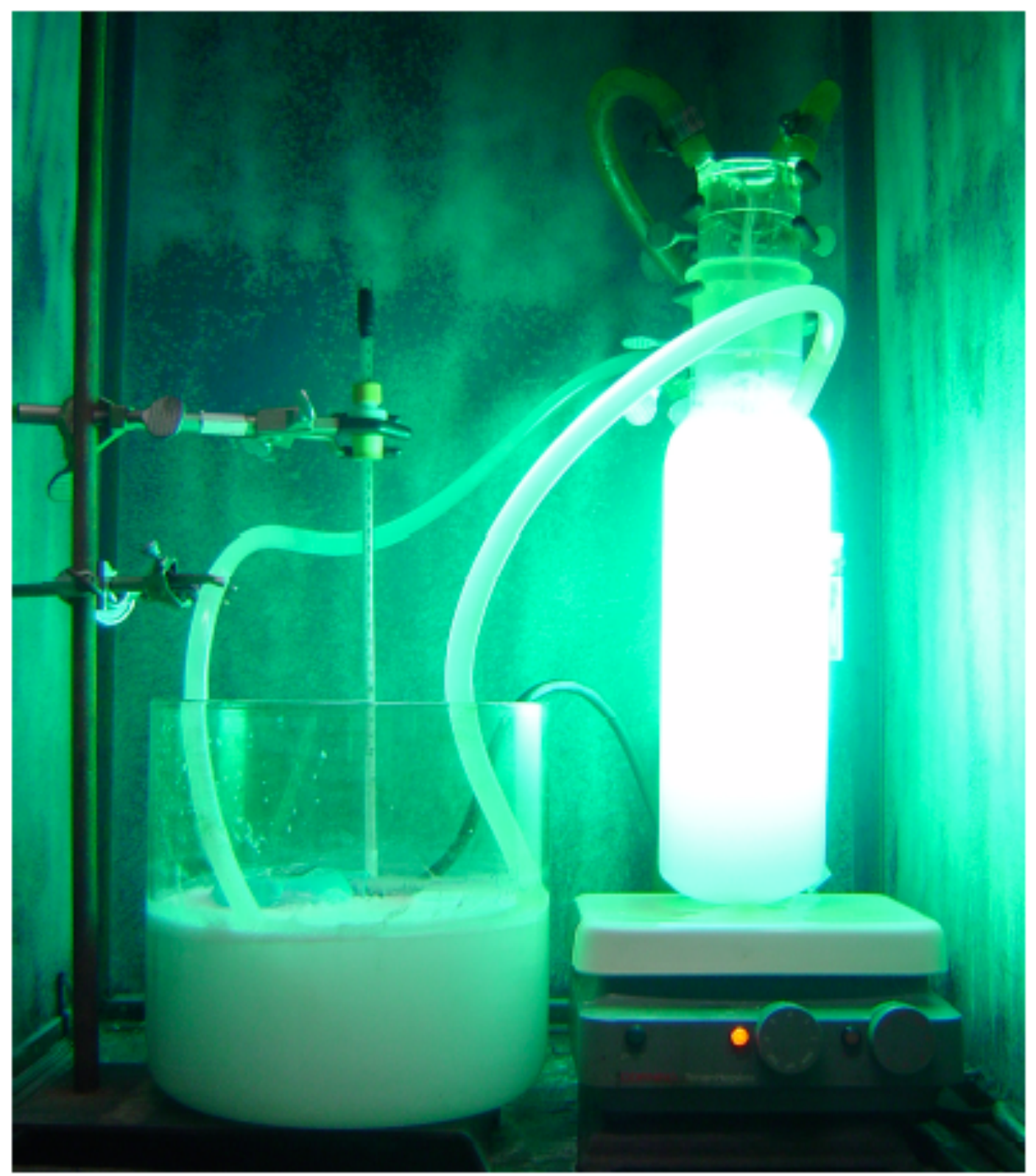

Figure S8. Irradiation of a crystal suspension in an immersion well using a flow system. 


\section{Particle Separation}

To separate the crystals from SDS, the suspension was filtered through a Whatman cellulose filter, grade 1. After filtration, water was used to wash all SDS from the crystals. Although the nominal particle size retention for the filter was $11 \mu \mathrm{m}$, most of the particles stayed in the filter.

\section{Quantum Yield Measurements ${ }^{5}$}

Argon-bubbled benzene solutions and crystal suspension were irradiated in Pyrex tubes through $0.002 \mathrm{M} \mathrm{K}_{2} \mathrm{CrO}_{4}$ in $1 \%$ aqueous $\mathrm{K}_{2} \mathrm{CO}_{3}$ (maximum transmission, $313 \mathrm{~nm}$ ) with the $450 \mathrm{~W}$ Hanovia medium pressure mercury lamp. ${ }^{6}$ The quantum yields were determined with reference to the disappearance of valerophenone in benzene as actinometer. The quantum yield of valerophenone disappearance in benzene was taken as $0.35 .^{7}$ The conversion of valerophenone was kept under $10 \%$, in the range where conversion is linear as a function of irradiation time. The quantum yields are reported in the table are reproducible within \pm 0.004 .

\begin{tabular}{|c|c|c|}
\hline & $\Phi_{\text {cons }}$ & $\Phi_{\mathrm{DCK} \rightarrow \mathrm{DC}}$ \\
\hline Actinometry $^{\mathrm{a}}(313 \mathrm{~nm})$ & & \\
\hline hexanes & 0.50 & 0.07 \\
\hline benzene & 0.41 & 0.20 \\
\hline crystal suspension & 0.19 & 0.19 \\
\hline
\end{tabular}

5 Wagner, P. J.; Kochevar, I. E.; Kemppainen, A. E., "Type II Photoprocesses of Phenyl Ketones. Procedures for Determining Meaningful Quantum Yields" J. Am. Chem. Soc. 1972, 94, 7489-7494.

6 Murov, S. L.; Carmichael, I.; Hug, G. L. Handbook of Photochemistry; 2nd ed.; Marcel Dekker, Inc.: New York, NY, 1993.

7 Amrein, W.; Gloor, J.; Schaffner, K. Chimia 1974, 28, 185-188. 

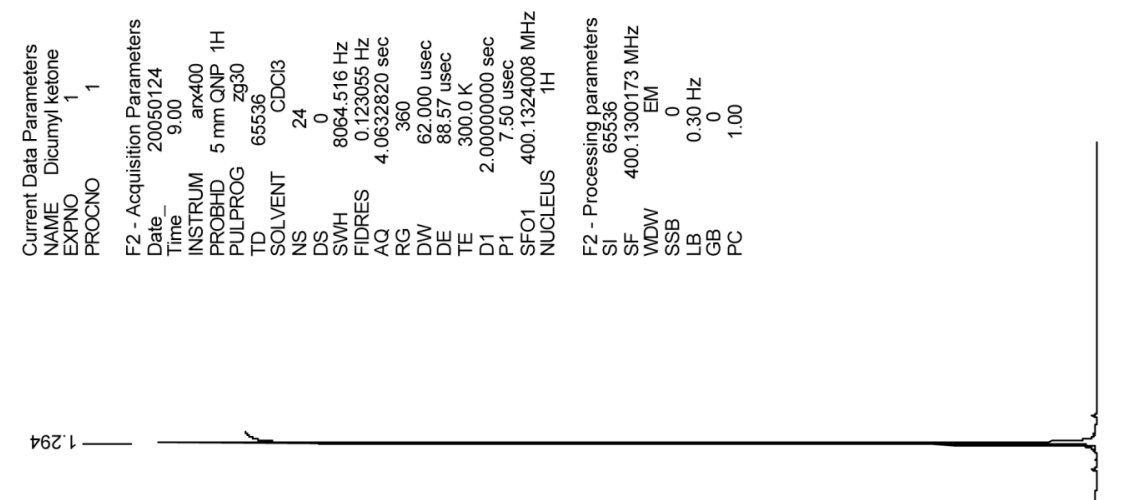

$+9$

$000^{\circ} \mathrm{Zl}$

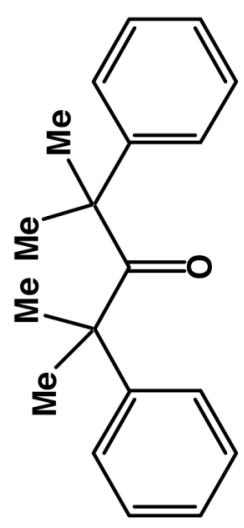

흠

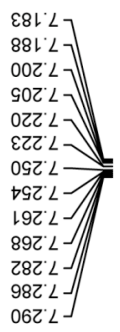

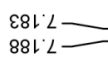

SOZ $L=$

$0 Z Z L=$

$\varepsilon Z Z \subset<$

${ }_{\triangle \rightarrow C Z}^{0} L=$

L9Z L

$89 Z L$

Z8Z' $L$

$062 L$

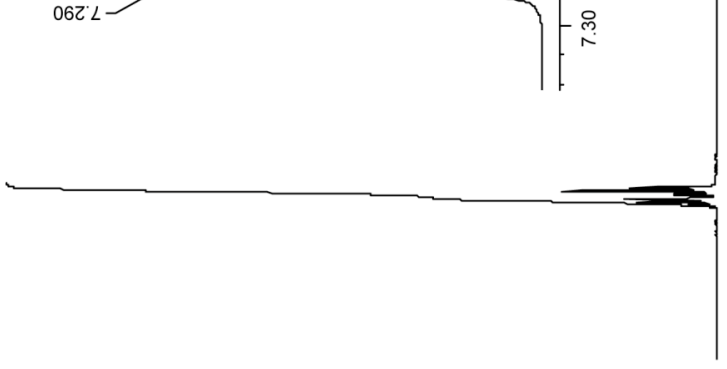

$\stackrel{\leftrightarrow}{0}$

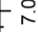

Figure S9. ${ }^{1} \mathrm{H}$ NMR spectrum of dicumyl ketone (DCK) 

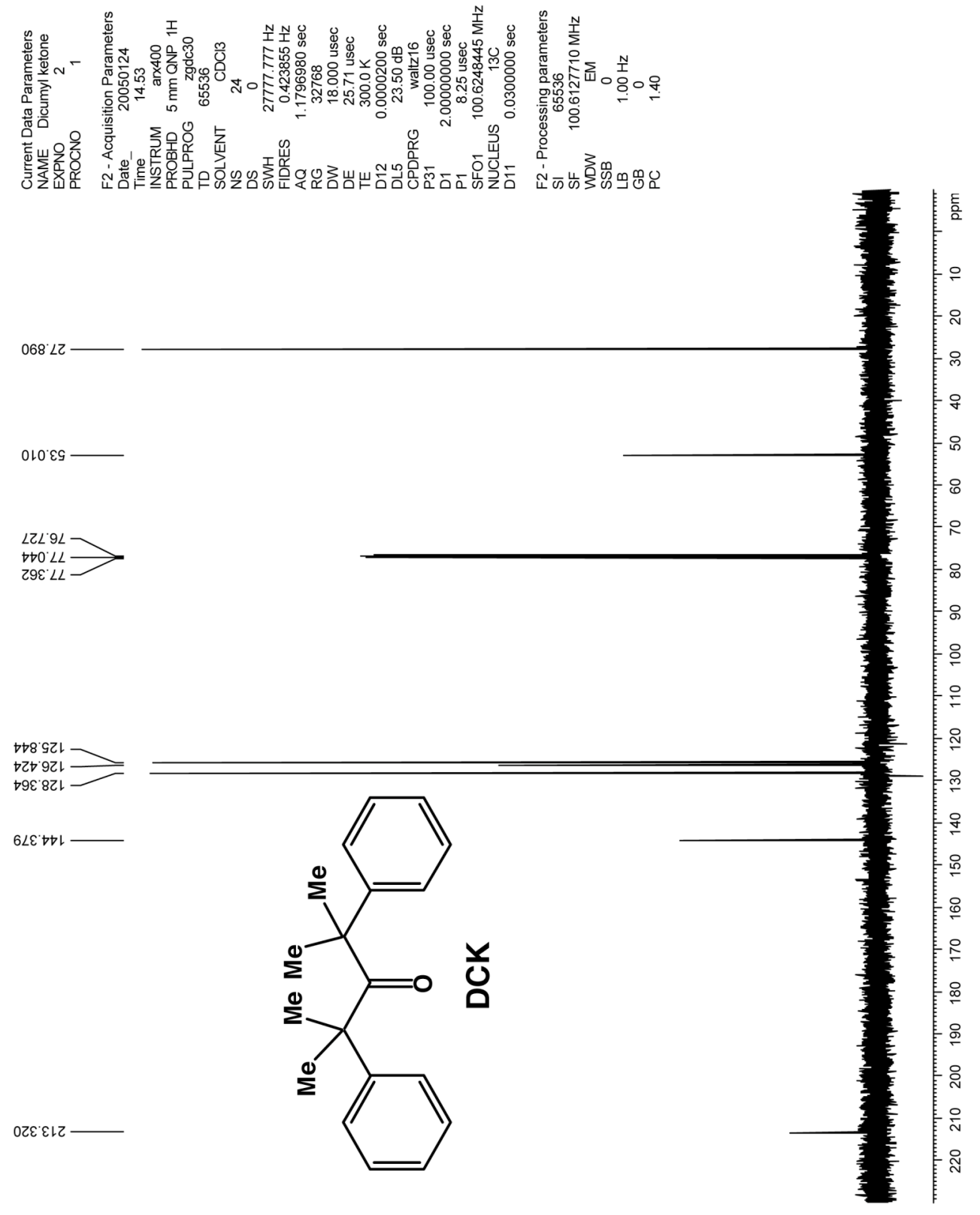

Figure S10. ${ }^{13} \mathrm{C}$ NMR spectrum of dicumyl ketone (DCK) 

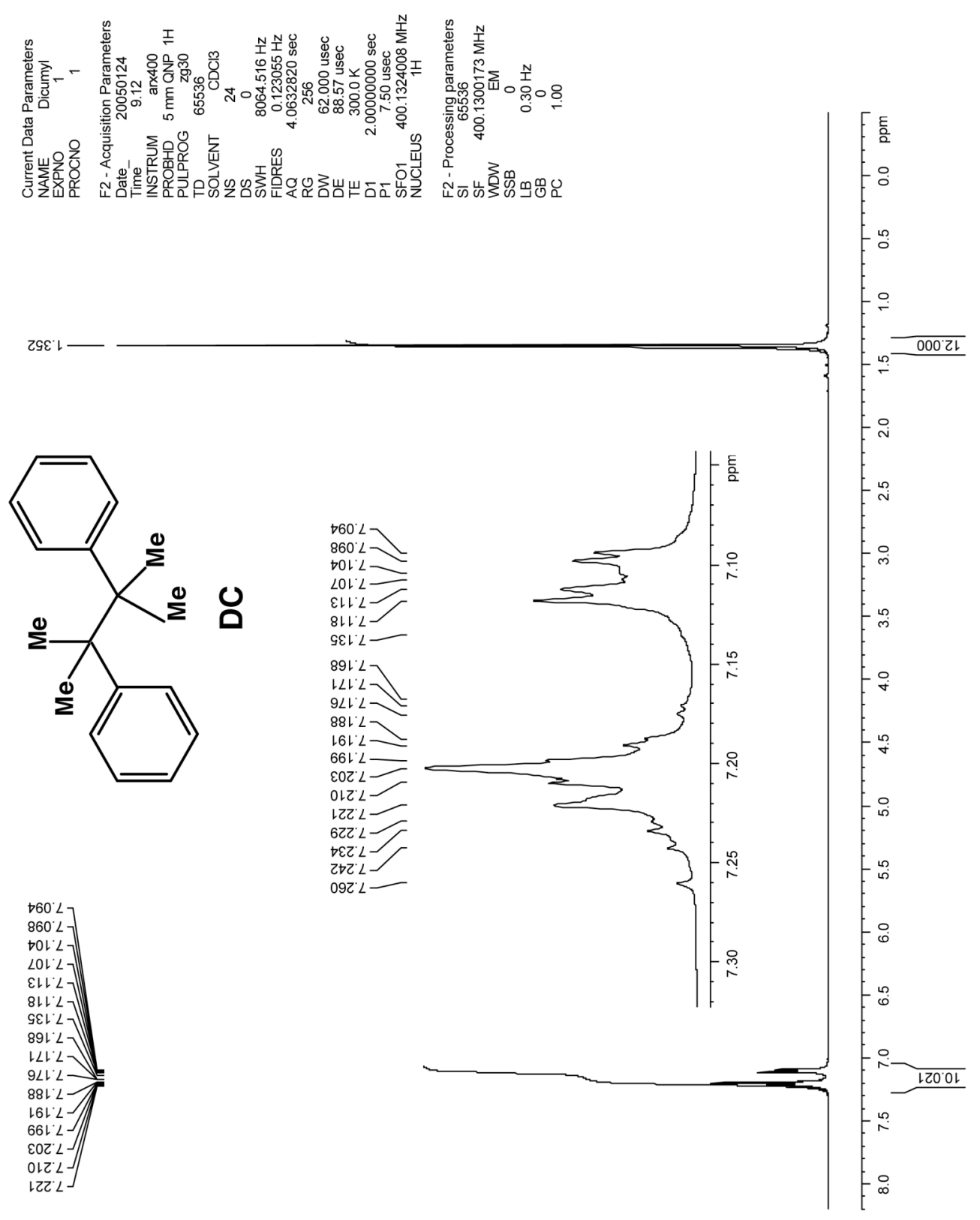

Figure S11. ${ }^{1} \mathrm{H}$ NMR spectrum of dicumene (DC) 

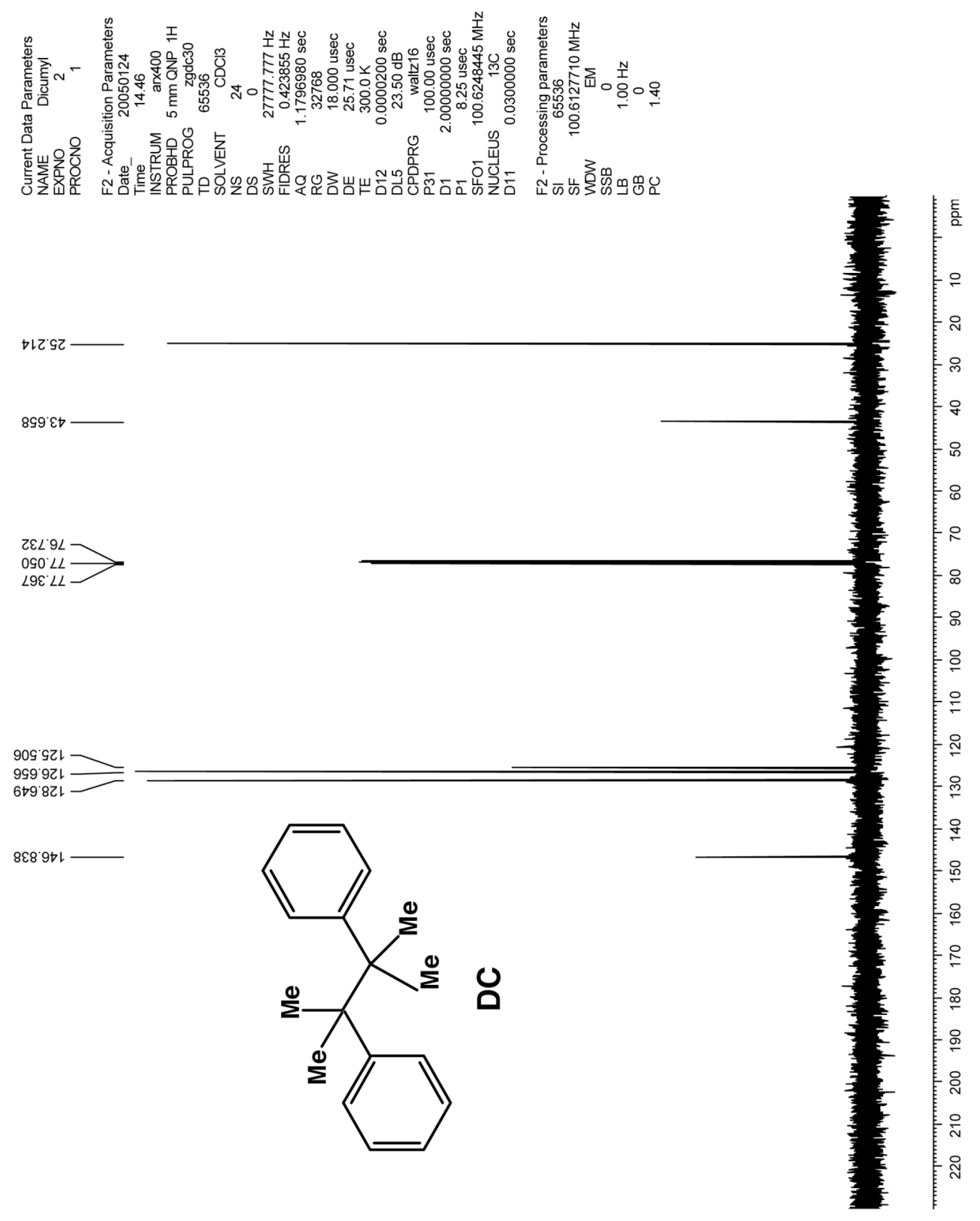

Figure S12. ${ }^{13} \mathrm{C}$ NMR spectrum of dicumene (DC). 